\title{
AN INVESTIGATION OF THE PROTONATION STATES OF HUMAN LACTOFERRIN IRON-BINDING PROTEIN
}

\author{
Lilia Anghel \\ Institute of Chemistry of Academy of Sciences of Moldova, 3, Academiei str., Chisinau MD-2028, Republic of Moldova \\ e-mail: anghel.lilia@gmail.com
}

\begin{abstract}
In this study, the protonation states of ionizable groups of human lactoferrin in various conformations were investigated theoretically, at physiological $\mathrm{pH}$ (7.365). These calculations show that the transition of the protein from a conformation to another one is accompanied by changes in the protonation state of specific amino acid residues. Analysis of the $p K_{a}$ calculatons underlined the importance of participation of two arginines and one lysine in the opening / closing of the protein. In addition, it was found that the mechanism of iron release depends on the protonation state of TYR-192. Protonated state of this residue in the closed form of lactoferrin will trigger the opening of protein and release of iron ions.
\end{abstract}

Keywords: lactoferrin, ionizable residues, protonation, continuum electrostatics.

Received: May 2014/ Revised final: March 2015/ Accepted: April 2015

\section{Introduction}

Human lactoferrin is an iron-binding glycoprotein member of the transferrin family. This protein was firstly isolated from milk, where it was found in elevated concentrations. Later, according to Steijns and Hooijdonk [1], this protein was identified in various products of exocrine glands located in the gateways of respiratory, digestive and reproductive systems suggesting a role in the defense against invading pathogens.

The high affinity of lactoferrin for iron determines a great part of its biochemical properties. Iron is an essential element for life and is used by microorganism to survive and replicate [2]. It is also a key element for many pathogenic microorganisms. The iron uptake by lactoferrin will make this element inaccessible for pathogenic microorganisms, preventing them from further development. In unbounded state, iron catalyses the reaction of formation of free radicals [3]. In a living organism, free radicals will generate many other harmful biochemical processes [4]. Thus, iron acquisition by lactoferrin has an indirect role in the protection of living organism from oxidative stress.

Lactoferrin molecules reversibly bind ferric ions and therefore they can be found in the iron-free form (apolactoferrin) and associated with iron ions (hololactoferrin). Apolactoferrin has an open conformation whilst hololactoferrin has a closed conformation (see Figure 1).

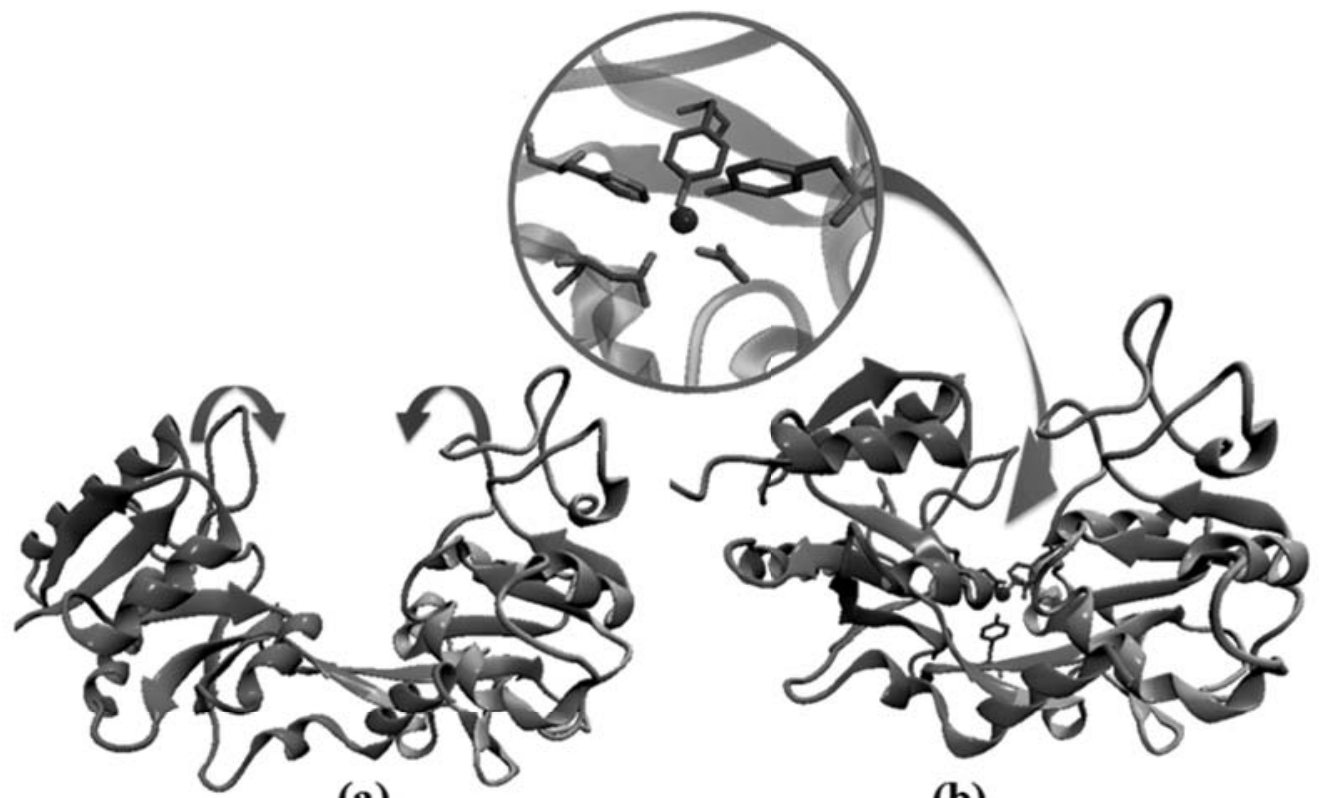

(a)

(b)

Figure 1. Graphical representation of the N-lobe of human lactoferrin open form (a) and closed holo-form (b). The position of carbonate and iron ions in the closed form are indicated. $\mathrm{N}$-lobe structure is presented in ribbon, ferric ion is presented as van der Waals sphere and carbonate ion as sticks. 
The X-ray crystallographic structure of this glycoprotein, 1B0L Protein Data Bank (PDB) code [5], consists of a polypeptide chain of 691 amino acid residues. Its structure is folded in two globular lobes referred as $\mathrm{N}$-(residues 1-333) and C-(residues 345-691) lobes connected with a short helical peptide chain (residue 334-344). Each lobe is further divided into domains (NI and NII and CI and CII). Both lobes are able to bind one ferric ion. The iron binding sites are similar in both lobes and among members of transferrin family. The coordination of a ferric ion is produced via one carboxylate oxygen, two phenolate oxygens and one imidazole nitrogen from one aspartate, two tyrosines and one histidine, respectively. Ferric ion is stabilized in the protein binding site through two oxygens from carbonate ion. $\mathrm{X}$-ray high-resolution structures of lactoferrins [5-9] isolated from various species show a high degree of similarity, approximately $70 \%$ identity between their structures. And each of the family members has a high degree of internal similarity $\sim 40 \%$ of sequence identity between N-lobe and C-lobe $[10,11]$.

Experimental evidence [12] has shown that lactoferrin molecule binds tightly ferric ions and retains them until $\sim \mathrm{pH} 3.0$ this suggests that conformational changes induced by iron binding and release are $\mathrm{pH}$ sensitive processes. Thus, investigation of the titratable residues from the lactoferrin structure is an important step in the studies of the mechanistic aspects of iron binding and release by lactoferrin protein molecules. The goal of this study is to compare the protonation states of ionizable groups of human lactoferrin in various conformations at the same $\mathrm{pH}$ value. This should allow the identification of the key residues from the protein structure involved in the process of iron-binding.

\section{Methods and computational details}

Structure and biochemical properties of proteins depend critically on the protonation state of ionizable groups of protein residues. Thus, various theoretical methods to determine ionization constants of residues in proteins through $p K_{a}$ calculations have been developed. In the present study, the $p K_{a}$ calculations were performed using $\mathrm{H}++$ web server [13-15]. Bashford and Karplus [16] described the basic methodology that $\mathrm{H}++$ relies upon. The method consists in $p K_{a}$ calculations for relevant ionizable groups in the system based on continuum electrostatic model within the framework of the Poisson Boltzmann model [17, 18]. According to the continuum electrostatic model, the protein is assumed to have a structure and is modeled as low dielectric region, which is embedded in a high dielectric region that represents the solvent. Atoms from the protein structure are considered as fixed-point charges, which are used to represent the charge of amino acids. The mathematical model of this concept is presented elsewhere [19].

The X-ray high-resolution crystallographic structures of open apolactoferrin and closed hololactoferrin were used as starting structures for the $p K_{a}$ calculations. These structures had the Protein Data Bank codes of 1LFH [20] determined at $2.80 \AA$ resolution and 1B0L [5] determined at $2.20 \AA$ resolution, respectively. All water molecules present in both PDB structure files were removed, according to the protocol for conducting computational experiments using $\mathrm{H}++$ server [13].

$p K_{a}$ calculations were conducted for typical physiological conditions, $\mathrm{pH} 7.365$ and an ionic strength of 150 $\mathrm{mM}$. Dielectric constants of 4.0 and 80.0 were used for protein and solvent (water), respectively. The PDB structures were initially protonated using AMBER force field [21] assuming $\mathrm{pH}$ of 7.365. Protonation state of histidine was automatically deduced from the van der Waals and hydrogen bond pattern in crystal structure of lactoferrin.

Visual Molecular Dynamics Software (VMD) is a molecular visualization program for displaying, animating, and analyzing large biomolecular systems using 3-D graphics and built-in scripting [22]. The graphical representation depicted in Figure 1 was generated using VMD.

\section{Results and discussion}

Metal binding capacity of proteins is influenced by intramolecular electrostatic interactions. The electrostatic interactions within a protein molecule are induced by ionization (protonation) states of titratable groups of the amino acids present in the protein structure. The latter will depend on the group type, location within the protein molecule, ionization state of other titratable sites in the proximity and the $\mathrm{pH}$ and ionic strength of the surrounding solvent [13]. Investigation of the ionizable residues of human lactoferrin and determination of key residues that trigger the conformation changes during the iron binding and release were performed using $p K_{a}$ calculations.

Table 1 summarizes the measured $p K_{a}$ values for the ionizable groups of human lactoferrin in four conformations at physiological $\mathrm{pH}$. Only those residues for which there is a difference greater than one $p K$ unit between any two of the models are listed in Table 1.

The protonation probability $\langle x\rangle$ (see Eq.(1)) of an isolated amino acid site is algebraically equivalent to the Henderson-Hasselbalch $(\mathrm{HH})$ equation $[23,24]$ which describes the sigmoidal standard titration curve:

$$
\langle x\rangle=\frac{10^{p K_{a}-p H}}{1+10^{p K_{a}-p H}}
$$


In this case, $p K_{a}$ value is equal to the $\mathrm{pH}$ at which the protonation probability of the amino acid site is $1 / 2$. Therefore, the so-called $p K_{1 / 2}$ values are often used to describe the titration behavior. The $p K_{1 / 2}$ values are not directly related to the free energy of the proton uptake and therefore in the case when titration curves are not monotonic these values can be ill-defined [23]. Lactoferrin polypeptide contains 691 amino acid residues and therefore most of the titration curves of its amino acid sites will have nonsigmoidal shapes because of the multitude of intramolecular interactions. For a better assessment of the protonation state of lactoferrin molecule in various conformations, the intrinsic $p K_{a}$ value was taken into account; this is the $p K_{a}$ value that a specific amino acid site would have if all other titratable sites were in the reference state.

Data have been collected for the open apo-form of human lactoferrin. For the comparison, the $p K_{a}$ values for the same groups measured in the closed apo-form (the holo-form with iron and carbonate ions removed), closed apo-form with iron ions only and holo-form (with both carbonate and iron ions). In the X-ray high-resolution crystallographic structure of open lactoferrin (1LFH PDB entry), only N-lobe is open whilst C-lobe is closed, therefore further only residues from the N-lobe will be discussed as they could provide the necessary information about the protein conformation changes.

ASP-60 has a lower $p K_{a}$ value in all closed conformations. According to the structural information from the PDB file, ASP-60 has a hydrogen bond with LYS-301 in the open conform, in the closed conformation this bond is missing, this might explain these $p K_{a}$ shifts $\left(\Delta p K_{a}\right)$.

At physiological $\mathrm{pH}$ tyrosines are deprotonated in the closed conformations except TYR-92, which has a relative stable protonation state in all conformations, and TYR-192 which is protonated in the closed conformations. TYR-192 has a lower $p K_{a}$ value in the open form and elevated $p K_{a}$ values in all closed conformations. This indicates that deprotonated TYR-192 will favor the iron binding whilst protonated TYR-192 state will trigger the iron release. Protonated state of TYR-192 in the closed models is mostly caused by interactions with the residue ASP-60.

Table 1

$\mathbf{p K}_{\mathrm{a}}$ values calculated for various conformations of human lactoferrin.

\begin{tabular}{|c|c|c|c|c|}
\hline \multirow[b]{2}{*}{ Residue } & \multicolumn{4}{|c|}{$p K_{a}$} \\
\hline & $a$ & $b$ & $c$ & $d$ \\
\hline GLU-51 & 6.973 & 5.318 & 5.296 & 5.293 \\
\hline *ASP-60 & 3.154 & -0.405 & -0.055 & 0.020 \\
\hline TYR-65 & 14.139 & 12.972 & 12.986 & 12.977 \\
\hline TYR-72 & 12.500 & 11.444 & 11.436 & 11.434 \\
\hline GLU-80 & 3.016 & 4.147 & 3.967 & 3.980 \\
\hline ARG-89 & 11.040 & 9.989 & 9.696 & 9.722 \\
\hline HIS-91 & 4.670 & 4.387 & 3.462 & 3.476 \\
\hline *TYR-92 & 12.218 & 12.034 & 12.615 & 12.857 \\
\hline ARG-121 & 8.169 & 3.781 & 3.781 & 3.667 \\
\hline ARG-133 & 16.499 & 11.879 & 11.946 & 11.936 \\
\hline LYS-163 & 9.867 & 7.833 & 7.826 & 7.815 \\
\hline ARG-171 & 14.304 & 11.913 & 11.908 & 11.908 \\
\hline *TYR-192 & 9.463 & 12.315 & 13.265 & 13.687 \\
\hline ARG-210 & 9.781 & 5.317 & 5.945 & 6.065 \\
\hline GLU-211 & 5.808 & 4.472 & 4.741 & 4.806 \\
\hline ARG-224 & 11.022 & 12.412 & 12.408 & 12.349 \\
\hline TYR-227 & 16.134 & 14.127 & 14.145 & 14.152 \\
\hline LYS-237 & 8.276 & 9.728 & 9.727 & 9.708 \\
\hline LYS-243 & 10.328 & 9.117 & 7.868 & 7.868 \\
\hline *HIS-253 & 2.371 & -2.260 & -1.762 & -1.662 \\
\hline ARG-258 & 14.548 & 10.862 & 10.821 & 10.824 \\
\hline ASP-297 & 2.468 & 3.987 & 3.625 & 3.616 \\
\hline LYS-301 & 7.727 & 4.980 & 4.634 & 4.676 \\
\hline ASP-315 & 2.828 & 4.931 & 4.927 & 4.929 \\
\hline
\end{tabular}

$a-p K_{a}$ values computed for open apo-form of human lactoferrin;

$b-p K_{a}^{a}$ values computed for closed apo-form (the holo-form with iron and carbonate ions removed);

$c-p K_{a}$ values computed for closed apo-form with iron ions only;

$d-p K_{a}$ values computed for holo-form (with both carbonate and iron ions).

* amino acids involved in iron coordination. 
At physiological $\mathrm{pH}$, amino acid HIS-253 was found to have low $p K_{a}$ values in all protein conformations. In all closed forms $p K_{a}$ value shifts downwards with $4 p K$ units. According to PDB structure files, protein closure creates an environment that induces a deprotonation of histidine through electrostatic interactions with the amino acids from its proximity.

LYS-301 also undergoes a $p K_{a}$ shift in the transition from the open to the closed form of lactoferrin, indicating that the side chains of this amino acid undergo electrostatic interactions with other amino acids in the proximity.

All arginine residues, ARG-121, ARG-133, ARG-171, ARG-258 have elevated $p K_{a}$ in the open form, and lower $p K_{a}$ in all closed conformations. In comparison to other arginines, ARG-121 and ARG-210 suffer great $p K$ shifts $\left(\triangle p K_{a} \approx 4\right)$ in the transition from the open conformation to the closed at physiological $\mathrm{pH}$, indicating on their importance in the process of iron binding.

Figure 2 presents the plot of the total charge of the open and closed conformations of lactoferrin molecules as a function of $\mathrm{pH}$. The curves present typical shapes characteristic to the proteins of the transferrin family. As it is depicted in Figure 2, lactoferrin is a basic, positively charged protein with an isoelectric point of 9.48 (open apo-form) and 9.78 (closed holo-form). Experimentally determined isoelectric point for human lactoferrin varies between 8.40 and 9.00 $[25,26]$, this is in reasonable agreement with the computed values, confirming the correctitude of the $p K_{a}$ calculations.

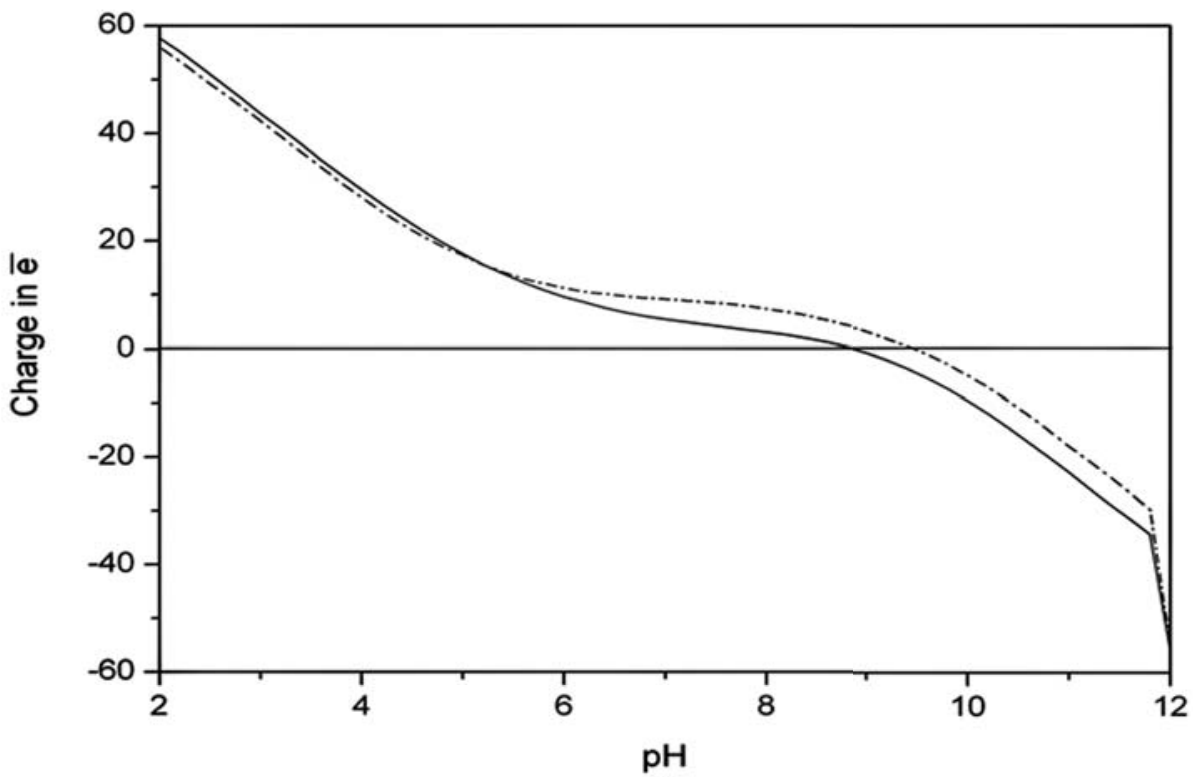

Figure 2. Plot of the total charge of the open apolactoferrin (solid line) and closed hololactoferrin (dashed line) as a function of $\mathrm{pH}$.

\section{Conclusions}

Human lactoferrin reversibly binds ferric ions, involving conformation changes of the protein state. Due to large intramolecular interactions, transition of the protein from the open to the closed form is accompanied by changes in the protonation states of protein residues. A continuum electrostatic model within the framework of the Poisson Boltzmann model was applied to investigate the protonation state of the protein residues at physiological $\mathrm{pH}$ in various protein conformations. These calculations confirm that the transition of the protein from a conformation to another is accompanied by changes in the protonation state of specific amino acid residues. In this sense, it was underlined the importance of participation of amino acids ARG-121, ARG-210, LYS-301 in the opening / closing of the protein. They also provided details about the mechanism of iron release, pointing on the importance of the protonation state of TYR192. Protonated state of this residue in the closed form of lactoferrin will trigger the opening of protein and release of iron ions.

\section{Acknowledgements} research.

I would like to acknowledge gratefully the supervision of Acad. Gheorghe Duca through each stage of my

I gratefully acknowledge the Program of the Romanian Plenipotentiary at JINR-Dubna (projects No.81/18.02.2013-37 and No.95/17.02.2014-26 within the JINR Theme No.04-4-1069-2009/2014) and the Moldovan State Programs (project No. 14.518.02.05A) for support. 


\section{References}

1. Steijns, J.M.; van Hooijdonk, A.C.M. Occurrence, structure, biochemical properties and technological characteristics of lactoferrin. British Journal of Nutrition, 2000, 84(1), pp. S11-S17.

2. Duca, Gh. Homogeneous Catalysis with Metal Complexes: Fundamentals and Applications. Springer Series in Chemical Physics: Berlin Heidelberg, 2012, XII, 478 p.

3. Duca, Gh.; Scurlatov, Yu.; Sychev, A. Redox Catalysis and Ecological Chemistry, Publishing Centre M.S.U.: Chisinau, 2002, 316 p.

4. Duca, Gh.; Secara, N. The free radicals in biological systems: formation and protection mechanisms. AKADEMOS: Journal of Science, Innovation, Culture and Art, 2010, 4(19), pp. 115-118.

5. Sun, X.L.; Baker, H.M.; Shewry, S.C.; Jameson, G.B.; Baker, E.N. Structure of recombinant human lactoferrin expressed in Aspergillus awamori. Acta Crystallographica, Section D, 1999, 55, pp. 403-407.

6. Karthikeyan, S.; Yadav, S.; Paramasivam, M.; Srinivasan, A.; Singh, T.P. Structure of buffalo lactoferrin at 3.3 A resolution at $277 \mathrm{~K}$. Acta Crystallographica, Section D, 2000, 56, pp. 684-689.

7. Moore, S.A.; Anderson, B.F.; Groom, C.R.; Haridas, M.; Baker, E.N. Three-dimensional structure of diferric bovine lactoferrin at $2.8 \AA$ resolution. Journal of Molecular Biology, 1997, 274, pp. 222-236.

8. Kumar, P.; Khan, J.A.; Yadav, S.; Singh, T.P. Crystal structure of equine apolactoferrin at $303 \mathrm{~K}$ providing further evidence of closed conformations of N and C lobes. Acta Crystallographica, Section D 2002, 58, pp. 225-232.

9. Khan, J.A.; Kumar, P.; Srinivasan, A.; Singh, T.P. Protein intermediate trapped by the simultaneous crystallization process. Crystal structure of an iron-saturated intermediate in the $\mathrm{Fe}^{3+}$ binding pathway of camel lactoferrin at 2.7 Å resolution. The Journal of Biological Chemistry, 2001, 276, pp. 36817-36823.

10. Baker, E.N.; Baker, H.M. Molecular structure, binding properties and dynamics of lactoferrin. Cellular and Molecular Life Sciences, 2005, 62, pp. 2531-2539.

11. Anghel, L. Lactoferrin: analysis of the structure profile. Chemistry Journal of Moldova, 2014, 9(2), pp. 99-106.

12. Baker, E.N.; Baker, H.M. Lactoferrin and iron: structural and dynamic aspects of binding and release. BioMetals, 2004, 17, pp. 209-216.

13. Gordon, J.C.; Myers, J.B.; Folta, T.; Shoja, V.; Heath, L.S.; Onufriev, A. H++: a server for estimating $\mathrm{pK}_{\mathrm{a}} \mathrm{s}$ and adding missing hydrogens to macromolecules, Nucleic Acids Research, 2005 1(33), pp. W368-W371.

14. Myers, J.; Grothaus, G.; Narayanan, S.; Onufriev, A. A simple clustering algorithm can be accurate enough for use in calculations of pKs in macromolecules, Proteins, 2006, 63, pp. 928-938.

15. Anandakrishnan, R.; Aguilar, B.; Onufriev, A.V. H++ 3.0: automating pK prediction and the preparation of biomolecular structures for atomistic molecular modeling and simulation, Nucleic Acids Research, 2012, 40(W1), pp. W537-W541.

16. Bashford, D.; Karplus, M. pKa of Ionizable Groups in Proteins: Atomic Detail from a Continuum Electrostatic Model. Biochemistry, 1990, 29, pp. 10219-10225.

17. Georgescu, R.; Alexov, E.; Gunner, M. Combining Conformational Flexibility and Continuum Electrostatics for Calculating $\mathrm{pK}_{\mathrm{a}} \mathrm{s}$ in Proteins. Biophysical Journal, 2002, 83, pp. 1731-1748.

18. Leimkuhler, B.; Chipot, C.; Elber, R.; Laaksonen, A.; Mark, A.; Schlick, T.; Schutte, C.; Skeel, R, Eds. New Algorithms for Macromolecular Simulation. Springer: Berlin Heidelberg, 2006, pp. 263-295.

19. Ullmann, G.M.; Kloppmann E.; Essigke, T.; Krammer, E.-M. Klingen, A.S.; Becker, T.; Bombarda, E. Investigating the mechanism of photosynthetic proteins using continuum electrostatics. Photosynthetic resources, 2008, 97, pp. 33-53.

20. Norris, G.E.; Anderson, B.F.; Baker, E.N. Molecular replacement solution of the structure of apolactoferrin, a protein displaying large-scale conformational change. Acta Crystallographica, Section B, 47, pp. 998-1004.

21. Ponder, J.W.; Case, D.A. Force fields for protein simulations. Advances in Protein Chemistry, 2003, 66, pp. 27-85.

22. Humphrey, W.; Dalke, A.; Schülten, K. VMD - Visual Molecular Dynamics. Journal of Molecular Graphics, 1996, 14, pp. 33-38.

23. Onufriev, A.; Case, D.A.; Ullmann, G.M. A Novel View of pH Titration in Biomolecule, Biochemistry, 2001, 40(12), pp. 3413-3419.

24. Cantor, C.R.; Schimmel, P.R. Biophysical Chemistry. Part III. The Behavior of Biological Macromolecules, W. H. Freeman and Co., New York, 1980, 597 p.

25. Farnaud, S.; Evans, R.W. Lactoferrin-a multifunctional protein with antimicrobial properties. Molecular Immunology, 2003, 40, pp. 395-405.

26. Moguilevsky, N.; Retegui, L.A.; Masson, P.L. Comparison of human lactoferrins from milk and neutrophilic leucocytes. Biochemical Journal, 1985, 229, pp. 353-359. 